\title{
FATIGUE IN REPEATED-SPRINT EXERCISE IS RELATED TO MUSCLE POWER FACTORS AND REDUCED NEUROMUSCULAR ACTIVITY
}

\author{
Alberto Mendez-Villanueva ${ }^{1,2}$, Peter Hamer ${ }^{1,3}$ and David Bishop ${ }^{1,4}$ \\ ${ }^{1}$ School of Human Movement and Exercise Science, \\ The University of Western Australia \\ ${ }^{2}$ ASPIRE, Academy for Sports Excellence, Doha, Qatar \\ ${ }^{3}$ School of Health Science, The University of Notre Dame (Australia) \\ ${ }^{4}$ Team Sport Research Group, Facoltà di Scienze Motorie, Università degli Studi di \\ Verona, Italy
}

\section{Current address for correspondence}

Alberto Mendez-Villanueva, $\mathrm{PhD}$

Performance Enhancement and Talent Identification Section

ASPIRE, Academy of Sports Excellence

P.O. Box 22287, Doha, Qatar

Fax: (+974) 4136190

Phone: (+974) 4136190

E-mail: jose.villanueva@aspire.qa 


\section{ABSTRACT}

Purpose: The purpose of this study was 1) to determine the relationship between each individual's anaerobic power reserve (APR) [i.e., the difference between the maximum anaerobic $\left(\mathrm{P}_{\text {ana }}\right)$ and aerobic power $\left(\mathrm{P}_{\text {aer }}\right)$ ] and fatigability during repeated-sprint exercise and 2) to examine the acute effects of repeated sprints on neuromuscular activity, as evidenced by changes in the surface electromyogram (EMG) signals. Methods: Eight healthy males carried out tests to determine $\mathrm{P}_{\text {ana }}$ (defined as the highest power output attained during a 6-s cycling sprint), $\mathrm{P}_{\text {aer }}$ (defined as the highest power output achieved during a progressive, discontinuous cycling test to failure) and a repeated cycling sprint test ( 10 x 6-s max sprints with $30 \mathrm{~s}$ rest). Peak power output (PPO) and mean power output (MPO) were calculated for each maximal 6-s cycling bout. Root mean square (RMS) was utilized to quantify EMG activity from the vastus lateralis (VL) muscle of the right leg. Results: Over the ten sprints, PPO and MPO decreased by $24.6 \%$ and $28.3 \%$ from the maximal value (i.e., sprint 1 ), respectively. Fatigue index during repeated sprints was significantly correlated with APR $(\mathrm{R}=0.87$; $\mathrm{P}<0.05)$. RMS values decreased over the ten sprints by $14.6 \%( \pm 6.3 \%)$. There was a strong linear relationship $\left(\mathrm{R}^{2}=0.97 ; \mathrm{P}<0.05\right)$ between the changes in MPO and EMG RMS from the vastus lateralis muscle during the ten sprints. Conclusion: The individual advantage in fatigue-resistance when performing a repeated sprint task was related with a lower anaerobic power reserve. Additionally, a suboptimal net motor unit activity might also impair the ability to repeatedly generate maximum power outputs.

Key words: Repeated sprints, muscle fatigue, anaerobic power reserve, EMG 


\section{INTRODUCTION}

According to the force-fatigability relationship, the greater the force exerted by a muscle or motor unit, during a given task, the more the muscle will fatigue (Enoka and Stuart 1992; Hunter and Enoka 2001). In repeated-sprint exercise, this relationship is also apparent. That is, individuals with high initial power levels usually experience the greatest power decrements (i.e., fatigue) (Hoylmard et al. 1987; Hamilton et al. 1991; Bishop et al. 2003; Wadley and Le Rossignol 1998; Bishop et al. 2004). The mechanisms underlying these differences in fatigability are unknown. Indirect evidence suggests that greater absolute forces are associated with an increased participation of anaerobic metabolism, resulting in a greater accumulation of metabolites (e.g., lactate, $\mathrm{H}+$ ) and/or earlier decrements in energy stores (e.g., PCr) (Hirvonen et al. 1987; Gaitanos et al. 1993; Bishop and Edge 2006). However, the finding that, despite being matched for single-sprint performance, trained subjects exhibit a greater fatigue resistance, with respect to untrained subjects, indicates that other mechanisms are involved in mediating the observed differences in performance decrements during repeated-sprint exercise (Bishop and Edge 2006).

Previous research may offer an explanation for the differences in muscle fatigability that occur in relation to the initial power during repeated-sprint exercise. It has been suggested that the same initial force level could be achieved by varying the participation of anaerobic and aerobic metabolism (Bishop and Edge 2006). An increased reliance on anaerobic metabolism to provide mechanical function is believed to result in impaired power retention during successive muscle contractions (Gaitanos et al. 1993; Balsom et al. 1994a, 1994b; Zhang et al. 2006). This might suggest that initial force-dependant decrements in repeated-sprint performance may be determined by the 
metabolic pathways supporting force production regardless of the absolute level of force generated. However, these assumptions remain untested. In part, this might be due to the inability to accurately measure whole-body anaerobic energy release. A recently proposed anaerobic reserve model, defined as the difference between a performer's maximum burst power and maximum aerobic power, might prove useful to study fatigue decrements in repeated sprints. The anaerobic reserve model estimates the portion of the power output that is provided anaerobically (Bundle et al. 2003; Weyand and Bundle 2005; Weyand et al. 2006) and is independent of the uncertainties associated with estimation of the anaerobic energy released during exercise (Bundle et al. 2003; Weyand and Bundle 2005). While a metabolic validation of this method is not currently available, the anaerobic reserve model might allow us to examine the relationship between anaerobic contribution to the first sprint and subsequent sprint decrement during repeated sprints.

Neuromuscular factors may also have an effect on muscle fatigue during repeated-sprint exercise. However, controversy exists regarding the role of neuromuscular factors on the etiology of muscle fatigue during repeated sprints. In order to maintain the desired power output during supramaximal sprints the nervous system would need to recruit all motor unit pools at their highest firing rate (James et al. 1995; Krustrup et al. 2004; Karatzaferi et al. 2001). Some studies have provided indirect support for this assumption showing a steady level of neural activation, as assessed via surface electromyogram (EMG) recordings, during repeated-sprint exercise despite mechanical performance becoming progressively impaired (Hautier et al. 2000; Billaut et al. 2005). In contrast, other investigations have found a clear attenuation of muscle activation, as assessed via muscle functional magnetic resonance imaging, in responses 
to maximum intermittent cycling sprints (Kinugasa et al. 2004) and a reduction in the central nervous system's drive to the active musculature (Mendez-Villanueva et al., 2007a; Racinais et al. 2007; Drust et al. 2005). A suboptimal motor unit activity might therefore, potentially play a role in the development of muscle fatigue during maximal, repeated efforts (Kawakami et al. 2000; Taylor et al. 2000; Nordlund et al. 2004; Racinais et al. 2007). The collection of EMG data in the present experiment would provide additional insights regarding the neuromuscular adjustments associated with repeated-sprint performance employing a similar exercise protocol previously used by others (Gaitanos et al. 1993; Kinugasa et al. 2004; Billaut et al. 2005; Billaut et al. 2006; Racinais et al. 2007).

We therefore undertook this study to test two hypotheses. First, we hypothesized that individuals with lower anaerobic power reserves, implying a greater reliance on aerobic metabolism to support mechanical outputs, would develop less fatigue during repeated-sprint exercise. Second, we hypothesized that power decrements would be accompanied by alterations in neuromuscular activity, as evidenced by changes in EMG activity.

\section{METHODS}

\section{Subjects}

Eight healthy males, recreationally active in various sports, volunteered to participate in this study. The subjects' characteristics were as follows (mean \pm SD): age $19.8 \pm 0.8 \mathrm{y}$, height $\mathbf{1 . 8 4} \pm \mathbf{0 . 0 6} \mathbf{~}$, mass $81.4 \pm 8.6 \mathrm{~kg}$. The exercise protocol and all possible risks and benefits associated with participation in the study were explained to each subject. Each subject provided written informed consent prior to participating in 
the study. Approval for the study's procedures was granted by the Institutional Research Ethics Committee.

\section{Experimental Overview}

All subjects came to the laboratory for seven exercise sessions. At least $48 \mathrm{~h}$ separated the exercise sessions. An initial laboratory visit was scheduled to obtain data on physical characteristics. Also, during this first session subjects were familiarized with the peak oxygen uptake $\left(\mathrm{V}_{2 p e a k}\right)$ assessment protocol. During the second, third, fourth and fifth visit, subjects performed a single 6-s sprint on the front-access cycle ergometer. Also during these four sessions subjects were familiarized with the main repeated-sprint exercise protocol. In the sixth session, subjects performed a graded exercise test $(\mathrm{GXT})$ to determine $\mathrm{\vee}_{2 \text { peak. }}$ In the last session, they performed the $10 \times 6$ s test of repeated-sprint ability (RSA). Subjects performed their trials at the same time of the day ( \pm 2 h) with the laboratory conditions being approximately $20^{\circ} \mathrm{C}$ and $50 \%$ relative humidity during all trials. The subjects were asked to follow their normal diet and to refrain from any form of intense physical activity for the $24 \mathrm{~h}$ prior to testing and to not eat within $3 \mathrm{~h}$ before each testing session.

\section{Ergometer}

An air-braked, front-access cycle ergometer (Repco, Melbourne, Australia) was used to conduct the tests. The ergometer was interfaced with an IBM-compatible computer system to allow for the collection of data for the calculation of power generated on each flywheel revolution and work performed during each individual sprint repetition (Lab-VIEW, National Instruments Corp., Austin, TX). The power output of the air-braked cycle ergometer is proportional to the cube of the flywheel 
velocity. Instantaneous work is expressed as the work done during a $0.2 \mathrm{~s}$ measuring epoch. Work done is then totalled over the trial period to determine work (J) and expressed relative to time to determine power (W). An optical sensor monitored the velocity of the flywheel at a sampling rate of 128 pulses per flywheel revolution. Before testing, the ergometer was dynamically calibrated on a mechanical rig across a range of power outputs (100-2000 W). Peak power output (PPO) and mean power output (MPO) were calculated for each maximal 6-s cycling bout. Power output was also calculated every second during sprint 1 and 10 to generate a composite power curve for every subject.

\section{Anaerobic Power Reserve (APR)}

APR is quantified as the difference between the maximal anaerobic power ( $\left.\mathrm{P}_{\text {ana }}\right)$ and the maximal aerobic power ( $\mathrm{P}_{\mathrm{aer}}$ ) (Bundle et al. 2003; Weyand and Bundle 2005). $\mathrm{P}_{\mathrm{ana}}$ and $\mathrm{P}_{\mathrm{aer}}$ are empirically determined quantities that are representative of the body's functional limits for burst and endurance performance respectively (Weyand et al. 2006).

$\mathbf{P}_{\text {ana: }}$ Peak power outputs were determined from the highest power output that subjects were able to generate during a 6-s all-out cycling sprint (Mendez-Villanueva et al. 2007b). Each subject performed a 6-s standing sprint on the front-access cycle ergometer on four separate occasions. The testing sessions were separated by a minimum of 1 day and a maximum of 7 days. Before each test, subjects performed a standardized warm-up comprising 4 min of cycling at 100-120 W, followed by 3 bouts of maximal standing start acceleration (approximately 2 s) and then 3 min of rest prior to performing the 6-s sprint. Subjects were instructed to perform an "all-out"' effort 
from the beginning of the test until instructed to stop. All sprints were performed from the same initial pedal position. Toe clips and heel straps were used to secure the feet to the pedals. Strong verbal encouragement was provided during each trial.

$\mathbf{P}_{\text {aer: }}$ Maximal aerobic power was determined during a graded exercise test (GXT) consisting of graded exercise steps (4-min stages), using an intermittent protocol (1-min passive rest between stages). The test commenced at $80 \mathrm{~W}$ and thereafter, intensity was increased by $30 \mathrm{~W}$ every 4 min until volitional exhaustion. Subjects were required to maintain the set power output, which was displayed on a computer screen in front of them. Peak aerobic power (i.e., $\mathrm{P}_{\mathrm{aer}}$ ) was taken as the highest power output achieved during the GXT. The test was stopped when the subject could no longer maintain the required power output. Strong verbal encouragement was provided to each subject as they came to the end of the test. During the GXT, expired air was continuously analyzed for $\mathrm{O}_{2}$ and $\mathrm{CO}_{2}$ concentrations using Ametek gas analyzers (Applied Electrochemistry, SOV S-3A11 and COV CD-3A, Pittsburgh, PA). Ventilation was recorded every 15 s using a turbine ventilometer (Morgan, 225A, Kent, UK). The gas analyzers were calibrated immediately before and verified after each test using three certified gravimetric gas mixtures (BOC Gases, Chatswood, Australia); the ventilometer was calibrated preexercise and verified postexercise using a 1-L syringe in accordance with the manufacturer's instructions. The ventilometer and gas analyzers were connected to an IBM PC that measured and displayed variables every $15 \mathrm{~s}$. $\dot{\mathrm{V}} \mathrm{O}_{2 \text { peak }}$ was determined to be the highest $\mathrm{v}_{2}$ measured during $15 \mathrm{~s}$.

\section{Repeated-Sprint Exercise}


The exercise protocol consisted of 10, 6-s sprints on a front-access cycle ergometer interspersed with $30 \mathrm{~s}$ of recovery. Before the test, subjects performed a standardized warm-up, comprising 4 min of cycling at a power of 100 to $120 \mathrm{~W}$, followed by three bouts of maximal standing-start acceleration (approx 2 s) and 3 min of rest before performing the main trial. In separate sessions (see Experimental Design) performance in a single 6-s cycling sprint test was recorded and was then used as the criterion score during the main trial. Subjects were instructed to perform an "all-out" effort from the beginning of the test until instructed to stop. During the first sprint, subjects were required to achieve at least $95 \%$ of their criterion score, as a check on pacing. All the subjects satisfied the $95 \%$ of the criterion score. Toe clips and heel straps were used to secure the feet to the pedals. Strong verbal encouragement was provided during each trial. All of the sprints were performed from the same initial pedal position with the right crank arm located $45^{\circ}$ forward to the vertical axis. During the subsequent 30-s rest period after each sprint subjects remained quietly seated on the ergometer. A fatigue index (FI) for PPO and MPO was calculated as the percentage decline from the first to the last sprint for each subject.

\section{Muscle EMG}

The EMG activity from the vastus lateralis (VL) muscle of the right leg was recorded via bipolar Ag-AgCl surface electrodes at an interelectrode distance of $20 \mathrm{~mm}$. We chose the VL muscle because it has been reported that total power output during 10 , 6-s cycling sprints separated by $30 \mathrm{~s}$ of passive rest (same task as in the present experiment) was significantly correlated with the activation of VL, as evaluated by muscle functional magnetic resonance imaging (Akima et al. 2004). Therefore, the VL muscle might be used as a valid measure of lower-body muscle activity in the present 
experimental conditions. Before placing the electrodes, the overlying skin was carefully prepared. The hair was shaved, the skin lightly abraded to remove the outer layer of epidermal cells and thoroughly cleansed with alcohol to reduce the skin-electrode interface impedance to below 2 K-ohms. Electrodes were fixed lengthwise, parallel to a line bisecting the proximal and distal tendons, over the middle of the muscle belly. The electrodes were taped down with cotton wool swabs to minimise sweat-induced interference. The EMG reference electrode was placed over the right iliac crest. To prevent movement artefact, wires between the electrodes and the computer were secured to the skin with adhesive tape. The EMG signal was amplified (x 1000) (P511, Grass Instrument Division, West Warwick, RI) and sampled at a rate of $2048 \mathrm{~Hz}$ using a custom-written data acquisition program (Lab-VIEW, National Instruments Corp., Austin, TX). Before sampling, the EMG signals were analogue band-pass filtered (highpass $10 \mathrm{~Hz}$, low-pass $1000 \mathrm{~Hz}$ ) to remove unwanted noise and possible movement artefacts in the low-frequency region and to eliminate aliasing and other artefact in the high-frequency region. The EMG data were recorded between the onset and the end of each 6-s sprint. EMG recording was initiated by a digital trigger coincident with the start of the 6-s sprint and data collection stopped by a digital signal at the end of the sprint. After additional high-pass filtering (at $20 \mathrm{~Hz}$ ) to eliminate movement artefact, the root mean square (RMS) of the signal was calculated from each sprint. For each sprint, the RMS was normalized to the first sprint value, which was assigned the value of $100 \%$.

\section{Statistical Analysis}

One-way (sprint number) ANOVA with repeated measures were used to determinate significant differences in each dependent variable over time. Significant F- 
values were followed by post hoc comparisons with sprint 1 using repeated t-test with Bonferroni correction. Changes in average power for each second during sprint 1 and 10 were analyzed using Student's paired t-test. Relationships between variables were examined by means of linear regressions and Pearson product-moment correlations were computed. Results are presented as mean \pm standard error (SE). Significance $a$ priori was accepted when $\mathrm{P}<0.05$. All statistical analyses were performed by using SPSS for Windows (version 13.0, Chicago, IL).

\section{RESULTS}

\section{Mechanical Power}

Maximal anaerobic power $\left(\mathbf{P}_{\text {ana }}\right)$ : The maximum mechanical power output attained during the 6-s single cycling sprint was $18.8 \pm 0.4 \mathrm{~W} \cdot \mathrm{kg}^{-1}$. Individuals values ranged from 17.2 to $20.1 \mathrm{~W} \cdot \mathrm{kg}^{-1}$.

Maximal aerobic power $\left(\mathbf{P}_{\text {aer }}\right)$ : The mean for the maximum mechanical aerobic power output was $3.8 \pm 0.1 \mathrm{~W} \cdot \mathrm{kg}^{-1}$ and the corresponding $\mathrm{VO}_{2 \text { peak }}$ value was $53.7 \pm 2.0$ $\mathrm{mL} \cdot \mathrm{kg}^{-1} \cdot \mathrm{min}^{-1}$. Individuals values ranged from 3.4 to $4.4 \mathrm{~W} \cdot \mathrm{kg}^{-1}$ and 47.9 to 62.0 $\mathrm{mL} \cdot \mathrm{kg}^{-1} \cdot \mathrm{min}^{-1}$ for $\mathrm{P}_{\mathrm{aer}}$ and $\mathrm{VO}_{2 \text { peak }}$, respectively.

Anaerobic power reserve (APR): The mean APR was $15.0 \pm 1.4 \mathrm{~W} \cdot \mathrm{kg}^{-1}$ with individual values ranging from 13.6 to $16.7 \mathrm{~W} \cdot \mathrm{kg}^{-1}$. On average, the maximum anaerobic power outputs attained during the 6-s cycling sprint were $5.0 \pm 0.2$ times higher than the maximum power outputs that could be supported by aerobic power (Fig. 1). 


\section{INSERT FIGURE 1}

\section{Repeated sprints}

The PPO and MPO values recorded during each of the 6-s sprints are displayed in Figure 2. The highest PPO $\left(18.8 \pm 0.4 \mathrm{~W} \cdot \mathrm{kg}^{-1}\right)$ and MPO $\left(15.0 \pm 0.4 \mathrm{~W} \cdot \mathrm{kg}^{-1}\right)$ were recorded during the first sprint. When compared with the first sprint, there was a significant decrease in PPO (5.8\%) and MPO (4.9\%) during the second sprint $(\mathrm{P}<$ 0.001). The range of the decrease among individuals was between 0.6 and $10.0 \%$ for PPO and 2.0 and $7.1 \%$ for MPO. The decrease in PPO and MPO after the first five sprints averaged $13.8 \%$ and $17.1 \%(\mathrm{P}<0.001)$, respectively. The range of decrease was from 5.6 to $23.2 \%$ and 10.1 to $29.8 \%$ for PPO and MPO respectively. Over the ten sprints PPO and MPO decreased by 24.1\% $(\mathrm{P}<0.001)$ and $27.7 \%(\mathrm{P}<0.001)$ from the maximal value, respectively, ranging from 11.1 to $37.9 \%$ for PPO and 17.1 to $42.1 \%$ for MPO.

\section{INSERT FIGURE 2}

A composite power curve derived from 1-s averages of power output for sprint 1 and 10 is shown in Figure 3. When compared with sprint 1, fatigue associated with the performance of our protocol led to a significant $(\mathrm{P}<0.05)$ decrease in power output at each second of the power-time curve.

\section{INSERT FIGURE 3}

\section{EMG activity}


The changes in the EMG amplitude (RMS) across the repeated sprints are displayed in Figure 4. When compared with the first sprint, the decrease in RMS after the first five sprints averaged 9.2\% $(\mathrm{P}<0.01)$. The range of this decrease was from 3.6 to $21.8 \%$. Normalized RMS values decreased significantly over the ten sprints (14.3\%; $\mathrm{P}<0.001$ ). The range of the decrease among individuals was between 5.3 and $25.8 \%$.

\section{INSERT FIGURE 4}

\section{Relationship between variables}

The decrease in peak power output (W) (i.e., FI) over time (between sprint 1 and 10) was inversely related to the initial peak power output (W) (i.e., first sprint) ( $r=$ 0.80; $\mathrm{P}<0.05)$. FI during repeated sprints was also correlated with APR $(\mathrm{r}=0.87 ; \mathrm{P}<$ 0.05). FI was negatively correlated with $\mathrm{P}_{\mathrm{aer}}\left(\mathrm{W} \cdot \mathrm{kg}^{-1} ; \mathrm{r}=-0.65 ; \mathrm{P}<0.05\right)$. There was not significant correlation between $\mathrm{FI}$ and $\mathrm{VO}_{2 \text { peak }}\left(\mathrm{mL} \cdot \mathrm{kg}^{-1} \cdot \mathrm{min}^{-1} ; \mathrm{r}=-0.17 ; \mathrm{P}>0.05\right)$. There was a strong linear relationship $\left(\mathrm{R}^{2}=0.97 ; \mathrm{P}<0.05\right)$ between the changes in MPO and EMG RMS from the vastus lateralis muscle during the ten sprints (Figure 5).

\section{INSERT FIGURE 5}

\section{DISCUSSION}

The main finding was that subjects with higher anaerobic power reserves, implying a greater reliance on anaerobic processes to supply energy, recorded larger power decrements across the ten sprints. In addition, power decrements (i.e., fatigue) observed during repeated-sprint exercise were accompanied by a decrease in the EMG amplitude of the vastus lateralis muscle. 


\section{Early fatigue during repeated sprints}

During repeated-sprint exercise, performance can be defined as the ability to repeat maximum power output after previous exercise. The loss of power in the present study, observed over the ten 6-s sprints with 30 s of recovery $(24.1 \%$ and $27.7 \%$ for PPO and MPO respectively), demonstrated the occurrence of muscular fatigue. Decrements in power output during the present study were evident after the first sprint (Fig. 2), showing that after only one 6-s sprint, $30 \mathrm{~s}$ of recovery was not enough to reproduce power output in a subsequent sprint. These results have implications in the context of multiple-sprint sports since short recovery periods, during short-term intermittent exercise, have been reported not only to decrease muscular performance (e.g., running speed), but also to negatively affect the quality of specific sporting skills (Ferrauti et al. 2001). However, our results contrast with previous reports showing that a 30-s rest period is enough to restore power output for three or four consecutive 6-s sprints (Gaitanos et al. 1993; Balson et al. 1994b) or two 8-s sprints (Billaut et al. 2003). These contrasting results may be linked to differences in initial (i.e., first sprint) power output, subjects' training status or to the different ergometers employed (Hunter and Enoka 2001; Bishop et al. 2003; Bishop and Spencer 2004).

\section{Muscle power factors and fatigability were related}

In accordance with previous research (Holmyard et al. 1987; Hamilton et al. 1991; Bishop et al. 2003), the decline in power output (i.e., FI) over the first ten sprints was inversely related to the initial power output (i.e., the value recorded in the first 6-s sprint), indicating that $64 \%$ of the variance in fatigue index was explained by the initial power of our subjects. The underlying physiological mechanisms behind this association are unknown, but indirect evidence would suggest that greater absolute 
forces are associated with decrements in the power-generating capacity of the recruited fibres due to greater metabolite-induced disturbances (Gaitanos et al. 1993; Bishop and Spencer 2004, Nordlund et al. 2004). In this regard, it has been reported that subjects with better sprinting capabilities rely more on anaerobic metabolism to support mechanical function than subjects with lesser ability (Hirvonen et al. 1987; Gaitanos et al. 1993). The current understanding of skeletal muscle fatigue, for both whole-body and isolated muscle preparations, indicates that during intense sequential contractions the onset of fatigue has a metabolic basis (Westerblad et al. 2002; Zhang et al. 2006; Weyand and Bundle 2005; Weyand et al. 2006). Despite the exact mechanisms remaining unclear, available evidence suggests that a dependence on non-oxidative pathways of ATP resynthesis impairs muscle force production (Westerblad et al. 2002; Weyand et al. 2006; Zhang et al. 2006). Therefore, the greater fatigability observed in subjects with a high initial sprint performance may have been due, in part, to the subsequent greater metabolic disturbances, rather than solely due to their greater initial sprint performance per se.

\section{While metabolic contribution of different energy pathways was not directly} investigated in the current study, expressing fatigue decrements in muscle performance in relation to individual's APR allowed a novel examination of the relationship between initial power output and metabolic pathways supporting force production. The APR quantifies the difference between the maximum respective mechanical outputs supported by the anaerobic (i.e., $\mathrm{P}_{\text {ana }}$ ) and aerobic (i.e., $\mathrm{P}_{\text {aer }}$ ) power of the subject (Fig. 1) (Bundle et al. 2003; Weyand and Bundle 2005; Weyand et al. 2006). Hence, individuals with low anaerobic power reserves would have the capability to fuel a larger proportion of the active musculature with oxygen during all-out sprints 
(Weyand and Bundle 2005; Weyand et al. 2006). Accordingly, in the present study, subjects with lower anaerobic power reserves were better able to maintain muscle performance over the repeated sprints. These results might explain why several physiological adaptations related to an increased reliance on the aerobic metabolism to ATP resynthesis, such as greater mitocondria respiratory capacity (Thomas et al. 2004), higher maximal oxygen uptake (McMahon and Wenger 1998; Bishop et al. 2004; Tomlin and Wenger 2002), faster oxygen uptake kinetics (Dupont et al. 2005) or augmented blood-oxygen transporters (Balsom et al. 1994a), have been associated with an enhanced ability to resist fatigue during repeated sprints. Therefore, these results suggest that the performance of the musculoskeletal system may undergo fatiguedependent performance decrements dictated by the relative reliance on anaerobic metabolism to provide mechanical function regardless of the absolute level of force generated.

\section{Power output, EMG, and fatigue}

The neuromuscular activation of the contracting musculature is another physiological mechanism that can contribute to fatigue during repeated-sprint exercise. The power output and EMG amplitude (i.e., RMS) signal of the vastus lateralis declined in parallel throughout the contraction, despite the constant maximal effort of the participants for each sprint. This is in disagreement with previous research showing a steady level of neural activation during repeated-sprint exercise (Hautier et al. 2000; Billaut et al. 2005). Our results are, however, in agreement with those of Kinugasa et al. (2004) and Racinais et al. (2007), who reported a reduced muscle activation (assessed via muscle functional magnetic resonance imaging and surface electromyograms, respectively) when subjects performed the same exercise protocol as in the present 
experiment (i.e., 10 maximal cycling sprints with 30-s rest). Moreover, neural adjustments such as a reduction in the central nervous system's drive to the active musculature (Drust et al. 2005; Racinais et al. 2007) and alterations in neuromuscular activation of the contracting musculature (Billaut et al. 2005; Billaut et al. 2006) have also been previously reported.

While caution should be used when inferring motor control strategies from EMG (Farina et al. 2004), the fall in EMG activity observed in the present study might be related to the modulation of net motor-unit activity (i.e., progressive inhibition of motor units and/or the decrease in motor unit firing rate) (Farina et al. 2004). The decline in the EMG amplitude may also have been affected by impairments in neuromuscular propagation, in which the ability to repetitively conduct muscle fiber action potentials is diminished (Bellemare and Garzaniti 1988). Neuromuscular propagation failure, however, has been considered to be a less-likely possibility during repeated-sprint exercise (Racinais et al. 2007). As surface EMG is not able to distinguish between motor unit recruitment and rate coding, the precise mechanisms underlying the decrease in EMG activity cannot be ascertained. However, regardless of whether the decrease in EMG is due to rate coding, motor unit de-recruitment or both, our primary finding would be unaffected; the ability to reproduce muscle performance in subsequent sprints might be related with the ability of the nervous system to modulate motor unit activity.

Another candidate to explain the fall in power output during the present study might be the selective fatigue of fast-twitch fibres. These fibres dominate power production during supramaximal exercise (Karatzaferi et al. 2001), but are fatigued more easily than slow-twitch fibres (Barclay 1996), leaving slow-twitch fibres to govern 
subsequent power output. In our study, comparison of the average second-by-second power output over 6-s between the first sprint and sprint 10 showed that fatigue was accompanied by a downward shift in power during each one-second epoch from the first second onwards (Fig. 3). Given that subjects with a greater percentage of fast-twitch fibres are able to generate a higher force output (Hamada et al. 2003) selective fatigue of those fibres might be related with the failure to develop the expected power output in the present study. The proposed selective fatigue of fast-twitch fibres in the present study is further supported by the recorded EMG data. Fast-twitch fibres have higher twitch tensions, larger potentials, and faster conduction velocities than slow-twitch fibres. Thus, the recruitment of less fast-twitch fibres occurring towards the end of the exercise can be expected to be accompanied by a change in the recruitment pattern that would explain the decreases in EMG amplitude in the present study (Gerdle et al. 2001).

\section{Conclusive remarks}

The present study indicated that individuals with lower anaerobic power reserves, implying less reliance on anaerobic metabolism, showed a higher resistance to fatigue during repeated bouts of supramaximal cycling. These results suggest that the metabolic pathway supporting force production, and not the absolute level of force generated per se might explain power decrements during repeated sprints. Additionally, the associated decline in EMG amplitude would suggest that impaired repeated-sprint ability is also associated with a reduction in motor unit activity. Further work should be conducted to study the physiological trade-offs involved in the concurrent optimization of power and endurance, as physiological attributes that confer power are often

achieved at the expense of endurance capabilities (Rome and Lindstedt 1998). Such findings will provide a rationale for different exercise prescriptions and injury 
prevention and rehabilitation programs to maximize fatigue resistance of individuals whose performance outcomes are related to their ability to repeatedly perform highintensity intermittent exercise. 


\section{ACKNOWLEDGMENTS}

This study was supported by a grant from the Gatorade Sports Science Institute. The authors wish to express their sincere gratitude to all the participants for their maximal effort and cooperation. 


\section{REFERENCES}

Akima H, Kinugasa R, Kuno S (2004) Recruitment of the thigh muscles during sprint cycling by muscle functional magnetic resonance imaging. Int J Sports Med 25:18

Balsom P, Ekblom B, Sjodin B (1994a) Enhanced oxygen availablility during high intensity intermittent exercise decreases anaerobic metabolite concentration in blood. Acta Physiol Scand 150:455-456

Balsom PD, Gaitanos GC, Ekblom B, Sjodin B (1994b) Reduced oxygen availability during high intensity intermittent exercise impairs performance. Acta Physiol Scand 152:279-285

Barclay CJ (1996) Mechanical efficiency and fatigue of fast and slow muscles of the mouse. J Physiol 497:781-794

Bellemare F, Garzaniti N (1988) Failure of neuromuscular propagation during human maximal voluntary contraction. J Appl Physiol 64:1084- 1093

Billaut F, Basset FA, Falgairette G (2005). Muscle coordination changes during intermittent cycling sprints. Neurosci Lett. 380:265-269

Billaut F, Basset FA, Giacomoni M, Lemaître F, Tricot V, Falgairette G (2006) Effect of high-intensity intermittent cycling sprints on neuromuscular activity. Int $\mathrm{J}$ Sports Med 27:25-30

Billaut F, Giacomoni M, Falgairette G (2003) Maximal intermittent exercise: effects of recovery duration and gender. J Appl Physiol 95:1632-1637

Bishop D, Davis C, Edge J, Goodman C (2004) Induced metabolic alkalosis effects muscle metabolism and repeated-sprint ability. Med Sci Sports Exerc 36:807-813

Bishop D, Edge J (2006) Determinants of repeated-sprint ability in females matched for single-sprint performance. Eur J Appl Physiol 97:373-379 
Bishop D, Edge J, Goodman C (2004) The relationship between muscle buffer capacity and repeated-sprint ability in females. Eur J Appl Physiol 92:540-547

Bishop D, Lawrence S, Spencer M (2003) Predictors of repeated-sprint ability in elite female hockey players. J Sci Med Sport 6:199-209.

Bishop D, Spencer M (2004) Determinants of repeated sprint ability in well-trained team-sport and endurance-trained athletes. J Sports Med Phys Fit 44:1-6

Bundle MW, Hoyt RW, Weyand PG (2003) High-speed running performance: a new approach to assessment and prediction. J Appl Physiol 95:1955-1962

Drust B, Rasmussen P, Mohr M, Nielsen B, Nybo L (2005) Elevations in core and muscle temperature impairs repeated sprint performance. Acta Physiol Scand 183:181-190

Dupont G, Millet GP, Guinhouya C, Berthoin S (2005) Relationship between oxygen uptake kinetics and performance in repeated running sprints. Eur J Appl Physiol 95: $27-34$

Enoka RM, Stuart DG (1992) Neurobiology of muscle fatigue. J Appl Physiol 72:16311648

Farina D, Merletti R, Enoka RM (2004) The extraction of neural strategies from the surface EMG. J Appl Physiol 96:1486-1495

Ferrauti A, Pluim BM, Weber K (2001) The effect of recovery duration on running speed and stroke quality during intermittent training drills in elite tennis players. J Sports Sci 19:235-242

Gaitanos GC, Williams C, Boobis LH, Brooks S (1993) Human muscle metabolism during intermittent maximal exercise. J Appl Physiol 75:712-719 
Gerdle B, Karlsson S, Crenshaw AG, Elert J, Fridén J (2001) The influences of muscle fibre proportions and areas upon EMG during maximal dynamic knee extensions. Eur J Appl Physiol 81:2-10

Hamada T, Sale DG, MacDougall JD, Tarnopolsky MA (2003) Interaction of fibre type, potentiation and fatigue in human extensor muscles. Acta Physiol Scand 178:165173

Hamilton AL, Nevill ME, Brooks S, Williams C (1991) Physiological responses to maximal intermittent exercise: differences between endurance-trained runners and games players. J Sports Sci 9:371-382

Hautier CA, Arsac LM, Deghdegh K, Souquet J, Belli A, Lacour J-R (2000) Influence of fatigue on EMG/force ration and cocontraction in cycling. Med Sci Sports Exerc 32:839-843

Hirvonen J, Rehunen S, Rusko H, Harkonen M (1987) Breakdown of high-energy phosphate compounds and lactate accumulation during short supramaximal exercise. Eur J Appl Physiol 56:253-259

Hoylmard DJ, Cheetham ME, Lakomy HKA, Williams C (1987) Effects of recovery duration on performance during multiple treadmill sprints. In: Reilly T, Lees A, Davids K, Murphy WJ (ed). Science and Football. E \& F.N. Spoon, London, UK..

Hunter SK, Enoka RM (2001) Sex differences in the fatigability of arm muscles depends on absolute force during isometric contractions. J Appl Physiol 91:26862694

James C, Sacco P, Jones DA (1995) Loss of power during fatigue of human leg muscles. J Physiol 484:237-246

Karatzaferi C, de Haan A, van Mechelen W, Sargeant AJ (2001) Metabolism changes in single human fibres during brief maximal exercise. Exp Physiol 86:411-415 
Kawakami Y, Amemiya K, Kanehisa H, Ikegawa S, Fukunaga T (2000) Fatigue responses of human triceps surae muscles during repetitive maximal isometric contractions. J Appl Physiol 88:1969-1975

Kinugasa R, Akima H, Ota A, Ohta A, Sugiura K, Kuno S (2004) Short-term creatine supplementation does not improve muscle activation or sprint performance in humans. Eur J Appl Physiol 91:230-237.

Krustrup P, Soderlund K, Mohr M, Gonzalez-Alonso J, Bangsbo J (2004) Recruitment of fibre types and quadriceps muscle portions during repeated, intense kneeextensor exercise in humans. Pflugers Arch 449:56-65

McMahon S, Wenger HA (1998) The relationship between aerobic fitness and both power output and subsequent recovery during maximal intermittent exercise. J Sci Med Sport 1:219-227

Mendez-Villanueva A, Bishop D, Hamer P (2007a) Fatigue responses during repeated sprints matched for initial mechanical output. Med Sci Sports Exerc 39:2219-225.

Mendez-Villanueva A, Bishop D, Hamer P (2007b) Reproducibility of a 6-s maximal cycling sprint test. J Sci Med Sport 10:323-326.

Nordlund MM, Thorstensson A, Cresswell AG (2004) Central and peripheral contributions to fatigue in relation to level of activation during repeated maximal voluntary isometric plantar flexions. J Appl Physiol 96:218-225

Racinais S, Bishop D, Denis R, Lattier G, Mendez-Villanueva A, Perrey S (2007) Muscle deoxygenation and neural drive to the muscle during repeated sprint cycling. Med Sci Sports Exerc 39:268-274

Rome LC, Lindstedt SL (1998) The quest for speed: muscles built for high-frequency contractions. News Physiol Sci 13:261-268 
Taylor JL, Allen GM, Butler JE, Gandevia SC (2000) Supraspinal fatigue during intermittent maximal voluntary contractions of the human elbow flexors. J Appl Physiol 89:305-313

Thomas C, Sirvent P, Perrey S, Raynaud E, Mercier J (2004) Relationships between maximal muscle oxidative capacity and blood lactate removal after supramaximal exercise and fatigue indexes in humans. J Appl Physiol 97:2132-2138

Tomlin DL, Wenger HA (2002) The relationship between aerobic fitness, power maintenance and oxygen consumption during intense intermittent exercise. J Sci Med Sport 5:194-203

Wadley G, Le Rossignol P (1998) The relationship between repeated sprint ability and the aerobic and anaerobic energy systems. J Sci Med Sport 1:100-110

Westerblad H, Allen DG, Lannergren J (2002) Muscle fatigue: lactic acid or inorganic phosphate the major cause?. News Physiol Sci 17:17-21

Weyand PG, Bundle MW (2005) Energetics of high-speed running: integrating classical theory and contemporary observations. Am J Physiol 288:R956-R965

Weyand PG, Lin JE, Bundle MW (2006) Sprint performance-duration relationships are set by the fractional duration of external force application. Am J Physiol 290:R758-R765

Zhang S, Bruton JD, Katz A, Westerblad H (2006) Limited oxygen diffusion accelerates fatigue development in mouse skeletal muscle. J Physiol 572:551-559 


\section{FIGURE LEGENDS}

Figure 1. Maximum 6-s mechanical (full bar height, $\mathrm{P}_{\mathrm{ana}}$ ) and aerobic (height of the dark lower portion, $\mathrm{P}_{\mathrm{aer}}$ ) power output for each of the 8 subjects. The difference between these respective power outputs (the anaerobic power reserve, APR) is illustrated by the white upper portion of the bar. Numeric values for the ratio of $\mathrm{P}_{\mathrm{ana}} / \mathrm{P}_{\mathrm{aer}}$ appear within the bars of each subject. Data are expressed as watts per kilogram of body mass $(n=8)$.

Figure 2. Average peak power output (a) and average mean power output (b) for the entire group over the 10 sprints. Data are presented as mean \pm SE $(n=8)$. * Significantly lower $(\mathrm{P}<0.05)$ compared with sprint 1 .

Figure 3. Mean time course of second-by-second power output (W) (expressed in absolute values) during sprints 1 and 10 . Data are presented as mean \pm SE $(n=8) . *$ Significantly lower $(\mathrm{P}<0.01)$ compared with sprint 1 .

Figure 4. Normalised EMG amplitude (root mean square) changes of the vastus lateralis muscle for the entire group over the 10 sprints. Data are presented as mean \pm SE $(n=8) .{ }^{*}$ Significantly lower $(\mathrm{P}<0.05)$ compared with sprint 1.

Figure 5. Relationship between total work during sprints 1 to 10 (expressed as a \% of sprint 1) and vastus lateralis muscle EMG amplitude (i.e., RMS) (expressed as a \% of sprint 1 ) during the repeated-sprint exercise ( $\mathrm{n}=8$ for each data point). 
FIGURE 1

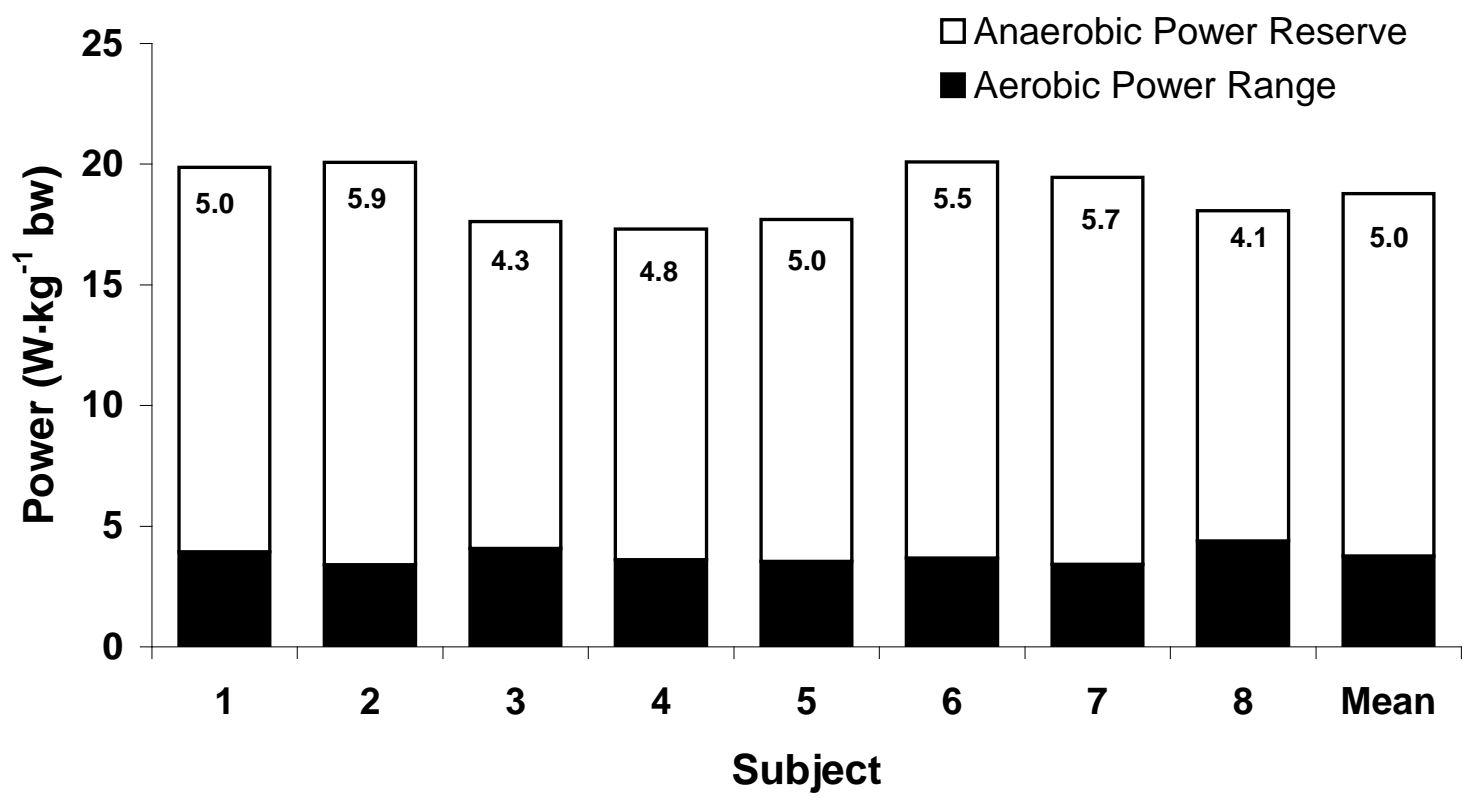


FIGURE 2

a

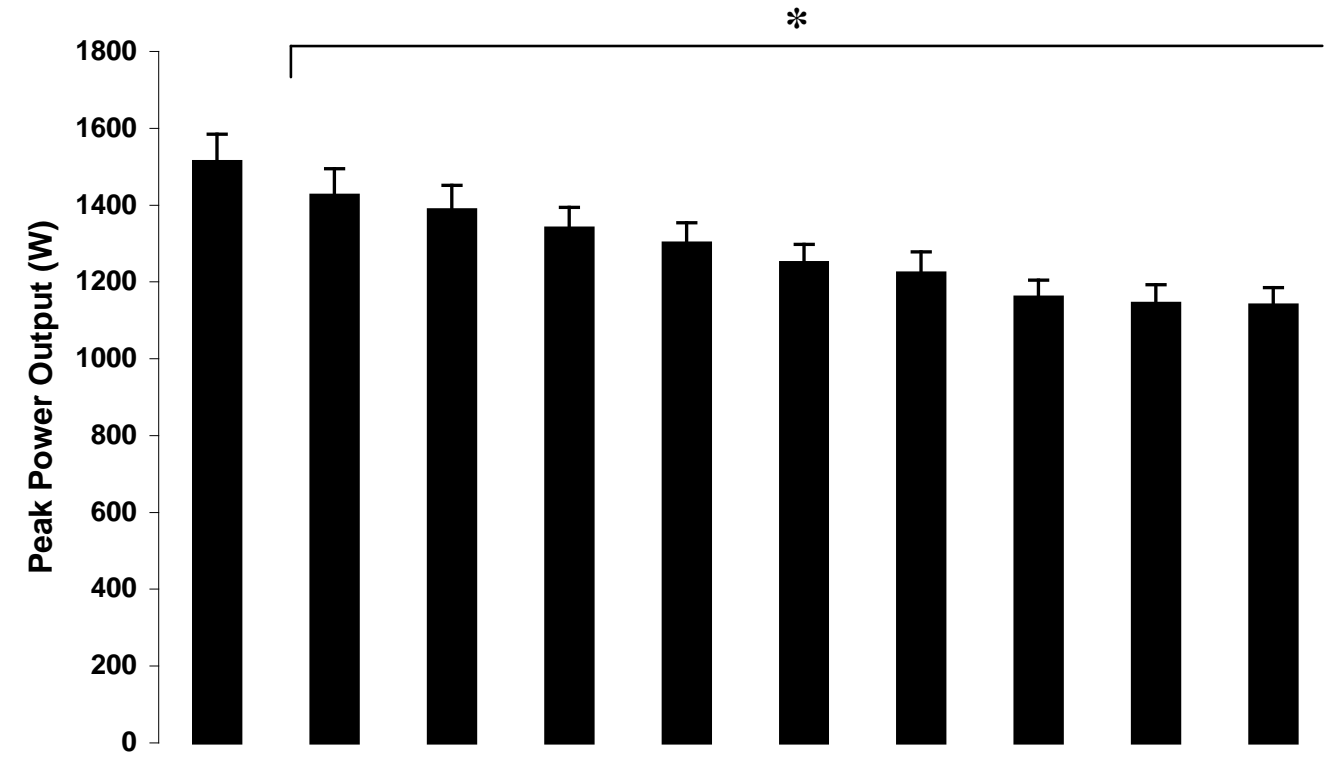

b

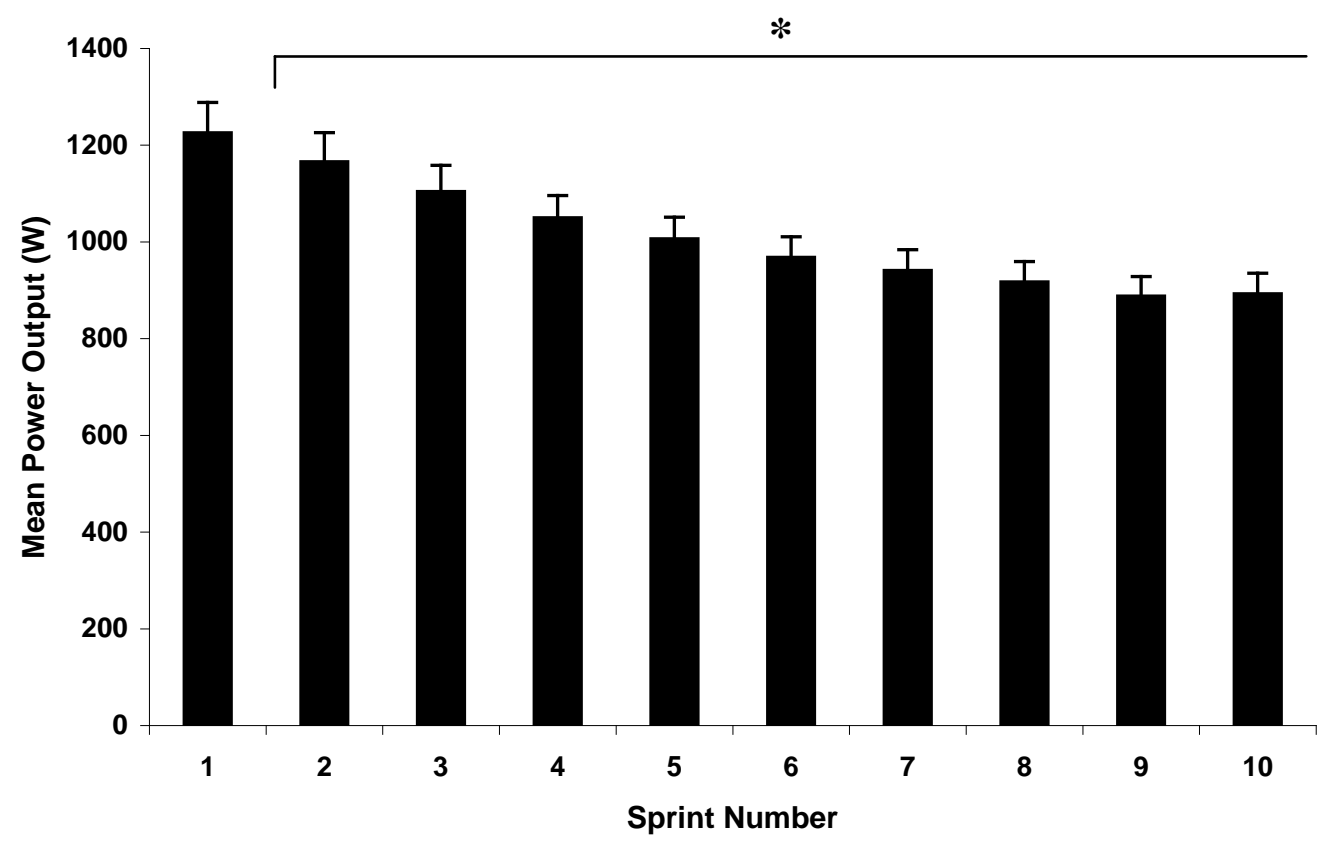


FIGURE 3

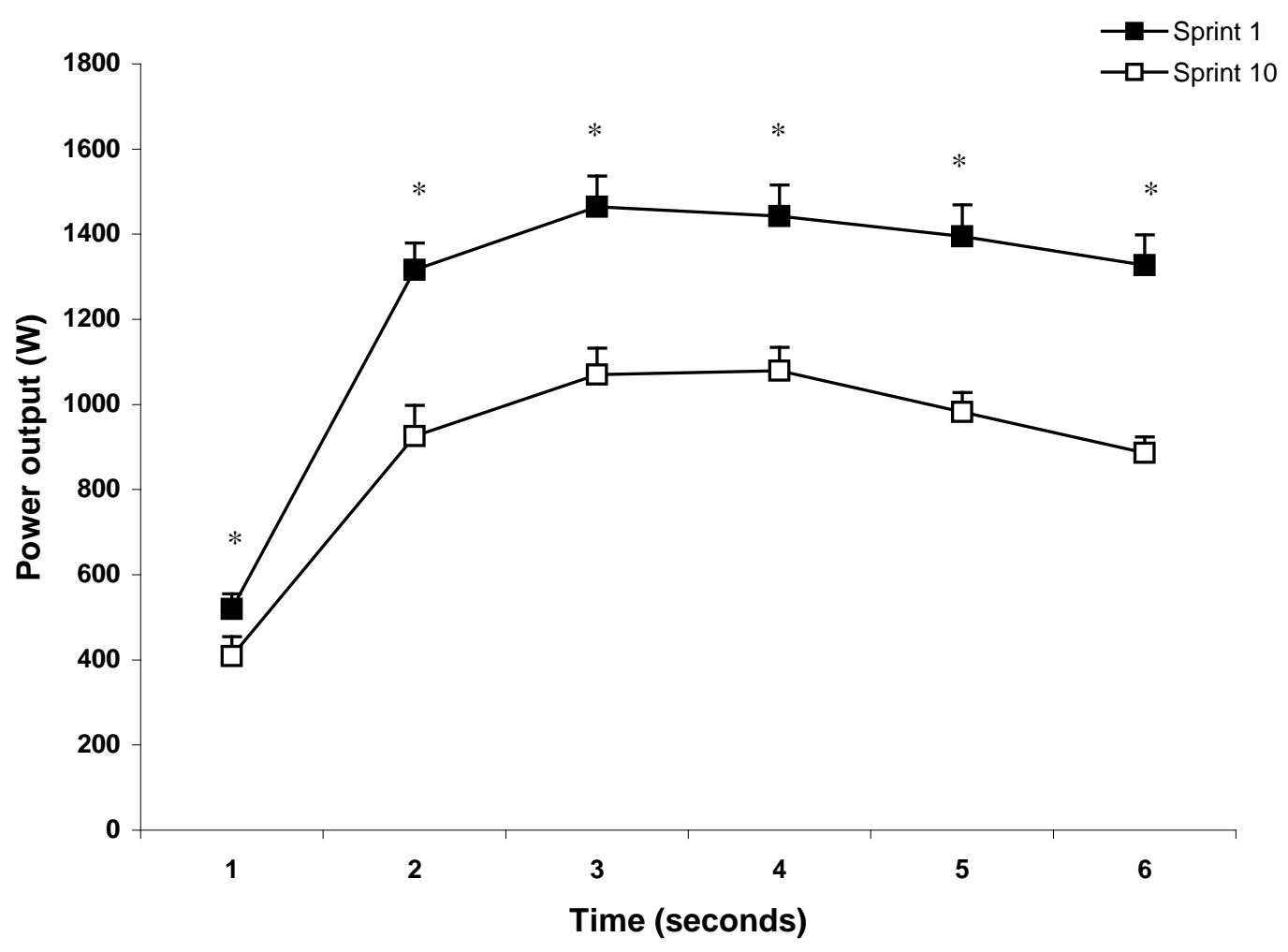


FIGURE 4

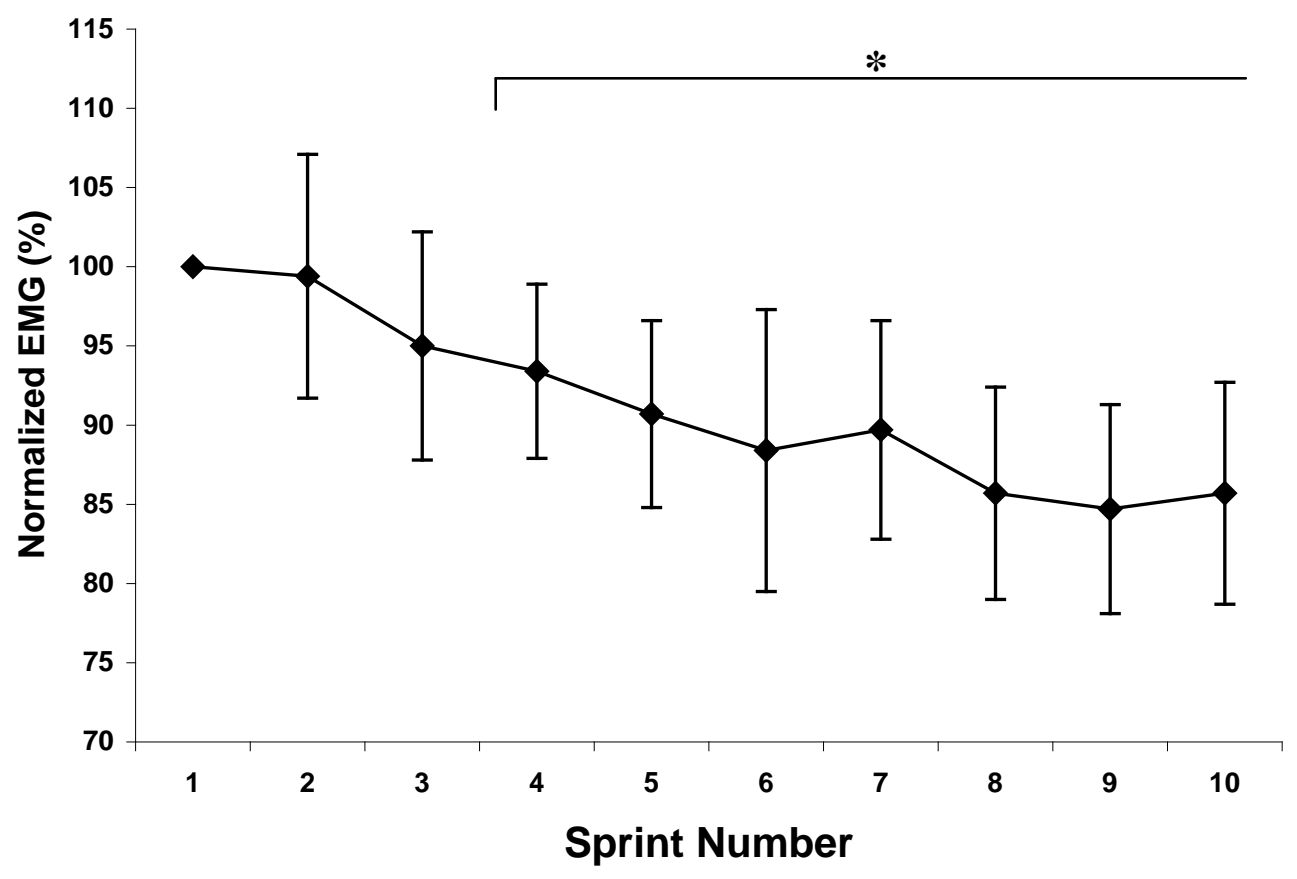


FIGURE 5

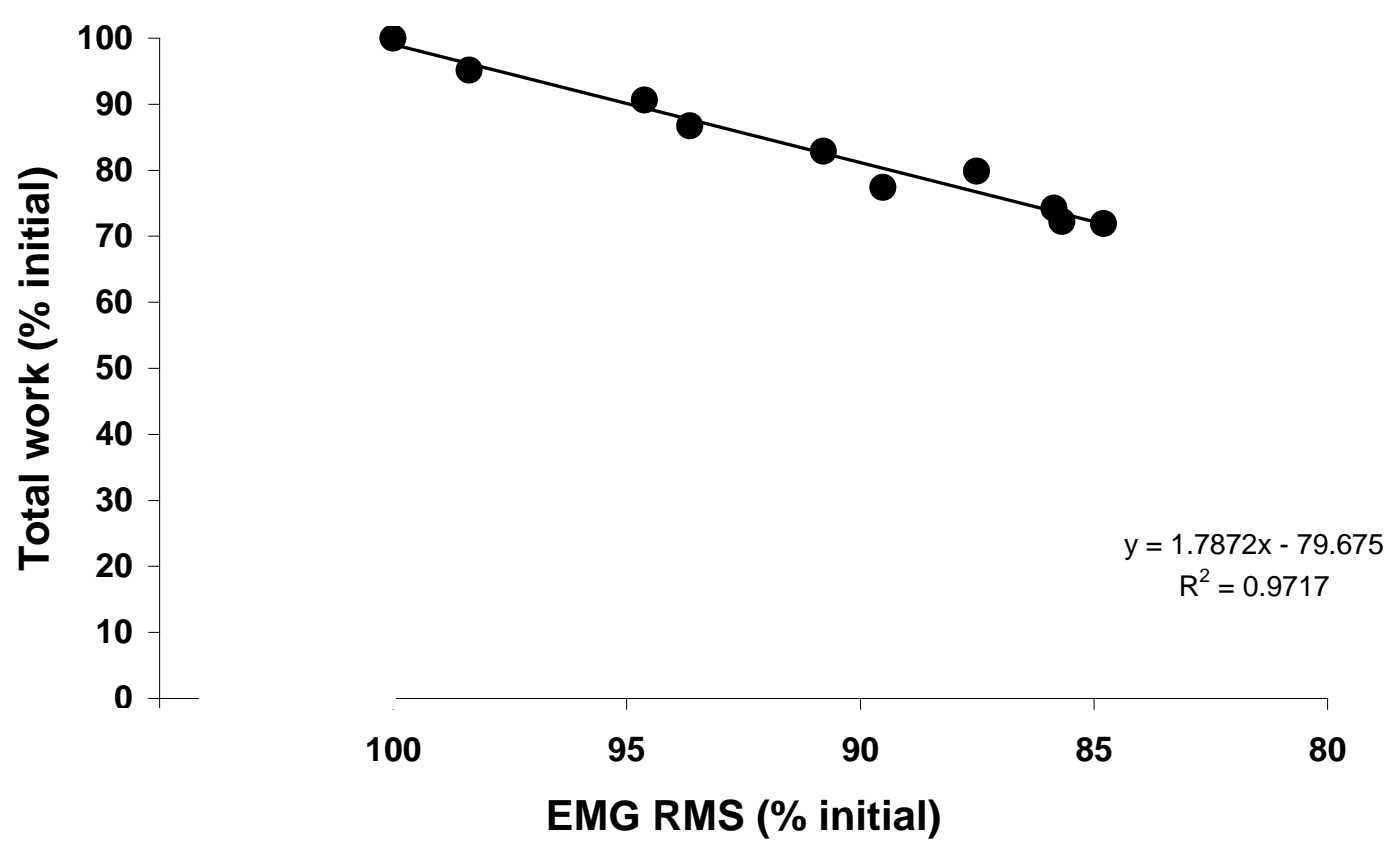

